\title{
THE GEOLOGIC EVOLUTION OF MARS: EPISODICITY OF RESURFACING EVENTS AND AGES FROM CRATERING ANALYSIS OF IMAGE DATA AND CORRELATION WITH RADIOMETRIC AGES OF MARTIAN METEORITES.
}

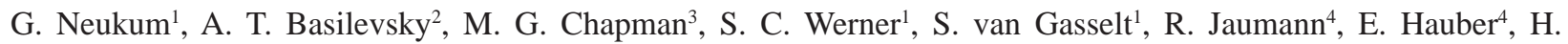
Hoffmann $^{4}$, U. Wolf ${ }^{4}$, J. W. Head ${ }^{5}$, R. Greeley ${ }^{6}$, T. B. McCord7, and the HRSC Co-Investigator Team

${ }^{1}$ Free University of Berlin, Inst. of Geosciences, 12249 Berlin, Germany (gneukum@zedat.fu-berlin.de), ${ }^{2}$ Vernadsky Inst. of Geochemistry and Analytical Chemistry, RAS, 119991 Moscow, Russia, ${ }^{3}$ U.S. Geological Survey, Flagstaff, AZ 86001, USA, ${ }^{4}$ DLR, Inst. for Planet. Expl., Rutherfordstrasse 2, 12489 Berlin, Germany, ${ }^{5}$ Brown University, Dept. of Geological Sciences, Providence, R.I. 02912, USA, ${ }^{6}$ Arizona State Univ., Dept. of Geological Sciences, Box 871404, Tempe, AZ 85287-1404, USA, ${ }^{7}$ Space Science Institute, Winthrop, WA 98862, USA.

Introduction: In early attempts of understanding the time-stratigraphic relationships on the martian surface by crater counting techniques and principles of stratigraphic superposition, most of the geological units and constructs came out as being rather old, in the range of billions of years [1-10]. On the other hand, most of the ages of the martian meteorites cluster at relatively young values of around 175 m.y., 300-600 m.y. and $1.3 \mathrm{Ga}$, whereas very few old ages $>3 \mathrm{Ga}$ had been found $[20,21]$.

The early cratering age data were based on post-Viking image data analysis. With the new data from MGS (MOC) [11], MEX (HRSC) [12,13], and Mars Odyssey (THEMIS) [14], it has become clear by now that the apparent discrepancy between the two age sets and the predominance of old ages was a selection effect due to the limited Viking resolution showing predominantly large, old features. Ages as young as a few 100s, a few tens or even a few million years have been determined since on the basis of the new high-res imagery with spatial resolutions in the meter to tens-of-meters range [1519]. It has become clear therefore by now, that there is no basic discrepancy with respect to the age ranges and occurrence of age groups per se.

Areas investigated, measurements: We reported on first preliminary results from investigation of a combination of HRSC and MOC imagery previously [22] where we believed to have seen peaks of activity temporally coinciding with martian meteorite age groups $[20,21]$. We have now been able to investigate a much higher number of areas and have in particular mapped out and analyzed for their geologic evolution and cratering ages two large outflow channel areas, Echus Chasma/Kasei Valles [23] and Mangala Valles [24]. In both areas we have found multistage geological histories with mixed volcanic, fluvial, glacial, and hydrothermal activity. The new data in combination with the previous data have been analyzed by way of a refined method of cratering age extraction [29] also giving fine details of periods of resurfacing from the characteristics of the measured crater size-frequency distributions as they deviate from the production size-frequency distri- bution due to resurfacing effects. This method was in a rudimentary form already applied by [5], refined by [19], and again refined in the course of our work [29]. In Fig. 1, examples of measurements showing such characteristic resurfacing effects and the way of extracting resurfacing ages is shown. As here, most martian crater size-frequency distributions do not follow the relatively steep production distributions at smaller crater sizes but show kinks and flatter distribution characteristics due to resurfacing and recratering events. There are only very few notable exceptions where the production function can be measured directly. This particular issue with the reconstruction of the function in pieces and for the few direct measurements of the undisturbed function over a larger size range has been treated by $[19,25]$ and the function put forward by Neukum and Ivanov [e.g., 6,17,18,26-28] has been confirmed in all aspects. This function is used in Fig. 1 in the way of isochrons for individual surface ages based on the Hartmann and Neukum [17] chronology.

Episodicity in the geologic evolution of the martian surface: In Fig. 2 the dating results for Echus/Kasei and Mangala on the basis of the mapping effort of $[23,24]$ and substantiated with a number of additional measurements on geologic constructs from all over the martian surface are compiled. All the ages from cratering measurements are compared with the radiometric ages of martian meteorites [21]. There is a striking appearance of peaking of the geological activity or episodicity of resurfacing at certain times: $\sim 3.5 \mathrm{Ga}, 1$ to 1.5 Ga, 300 to 600 m.y., 200 m.y. ago, respectively. Even more striking is that within relatively narrow limits, the cratering ages of the different age groups fall together with the age groups of martian meteorites. The martian meteorite ages reflect both igneous events and aqueous alteration events. So do the cratering ages. There is a remarkable paucity of age occurrences in the 2-3 Ga age range in the cratering data. This corresponds to a paucity of meteorite ages in the same, even somewhat more extended age range. This appears to be a hint to either lower geologic activity in this time frame, or, more likely, the covering up of more ancient activity by 
subsequent events $<2 \mathrm{Ga}$ ago, with the exception of the residues from the time $>3 \mathrm{Ga}$ ago (the peak at $\sim 3.5 \mathrm{Ga}$ ) when the martian surface was thoroughly shaped at a very high level of activity by gigantic volcanic, fluvial, and glacial events which could not be completely erased by later events.

Conclusion: We can demonstrate that there has been geologic activity on the martian surface in terms of volcanic, fluvial, glacial, hydrothermal activity at all times from $>4 \mathrm{Ga}$ ago until today. This activity must have declined in magnitude through time. This activity was not continual but episodic. The episodes we find on the martian surface in the crater frequency analyses are remarkably well coincident with the age groups of the martian meteorites found from radiometric dating. A very consistent picture of the evolution of the martian surface is shaping up in the comparison of two data sets of very different origin, telling us that Mars was geologically utterly active until 3-3.5 Ga ago but later has been resurfaced at a lesser and lesser rate making possible to identify different episodes of activity in many areas. The former apparent discrepancy between the "youthfulness" of martian meteorite ages and the oldappearing surface of Mars was an artifact from insufficient spatial resolution of the Viking imagery.

References: [1] Hartmann W. K. (1973) JGR, 78, 40964116. [2] Soderblom L. A. (1974) Icarus, 22, 239-263. [3] Neukum G. and Wise D. U. (1976) Science, 194, 1381-1387. [4] Hartmann W. K. et al. (1981) in: Basaltic Volcanism on the Terrestrial Planets, 1050-1125. [5] Neukum G. and Hiller K. (1981) JGR, 86, 3097-3121. [6] Neukum G. (1983) Habilitation Diss. LMU Munich, 186pp. [7] Tanaka K. L. (1986) JGR, 91, 139-158. [8] Tanaka K. L. et al. (1992) in: Mars, 345-382. [10] Strom R. G. et al. (1992) in: Mars, 383-423. [11] Malin M. C. et al. (1992) JGR, 97, 7699-7718. [12] Neukum G. et al. (2004) ESA SP-1240, 17-35. [13] Jaumann R. et al. (2007) PSS, in print. [14] Christensen P. R. (2002) AGU, Abstract \#P11B-07. [15] Hartmann W. K. (1999) Meteoritics \& Planet. Sci., 34, 167-177. [16] Hartmann W. K. and Berman D. C. (2000) JGR, 105, 15011-15026. [17] Hartmann W. K. and Neukum G. (2001) Space Sci. Rev., 96, 165-194. [18] Neukum G. et al. (2004) Nature, 432, 971-979. [19] Werner S. C. (2005) Doctoral Diss. FU Berlin. [20] Nyquist L. E. et al. (2001) Space Sci. Rev., 96, 55-86. [21] Borg L. and Drake M. J. (2005) JGR, 110, E12S03. [22] Neukum G. et al. (2006) LPSC XXXVII, Abstract \#2379. [23] Chapman M. G. et al. (2007) this volume. [24] Basilevsky A. T. et al. (2007) this volume. [25] Hartmann W. K. et al. (2007) Nature, submitted. [26] Neukum G. et al. (2001) Space Sci. Rev., 96, 55-86. [27] Neukum G. and Ivanov B. A. (1994) in: Hazards Due to Comets and Asteroids, 359. [28] Ivanov B. A. (2001) Space Sci. Rev., 96, 87-104. [29] Michael G. and Neukum G. (2007) this volume.

Acknowledgements: This work has been supported by the German Space Agency (DLR) and the German Science Foundation (DFG). Data reduction and editorial support by G. Michael, W. Zuschneid, and G. Mygiakis is acknowledged.
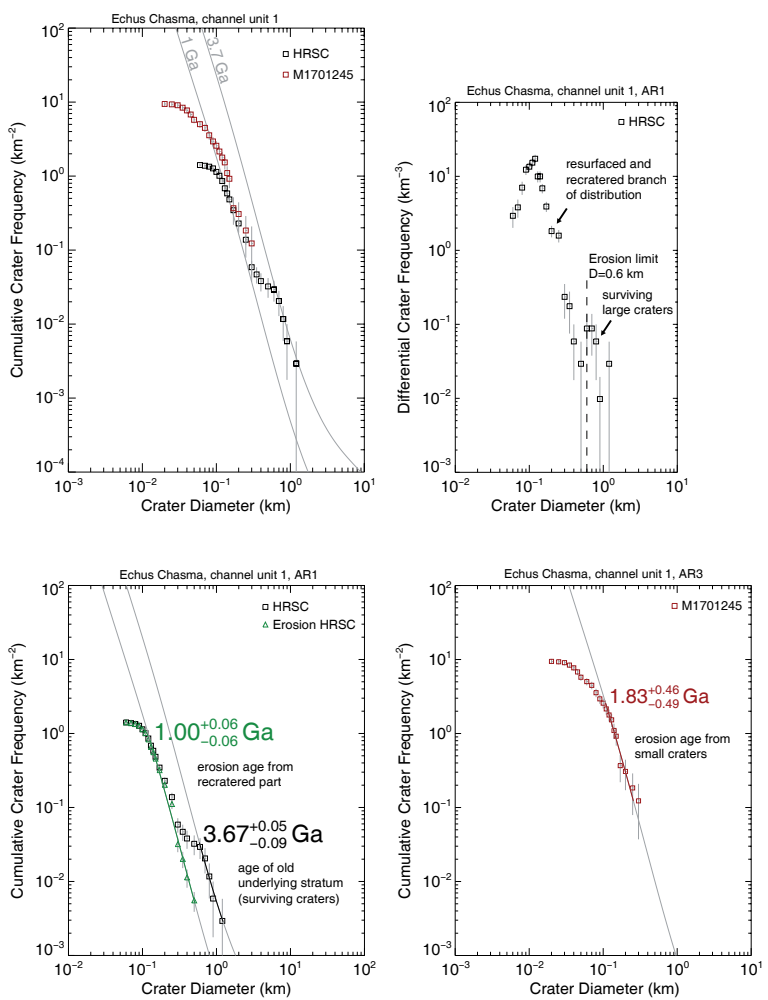

Fig. 1: Examples of measurements showing characteristic resurfacing effects.

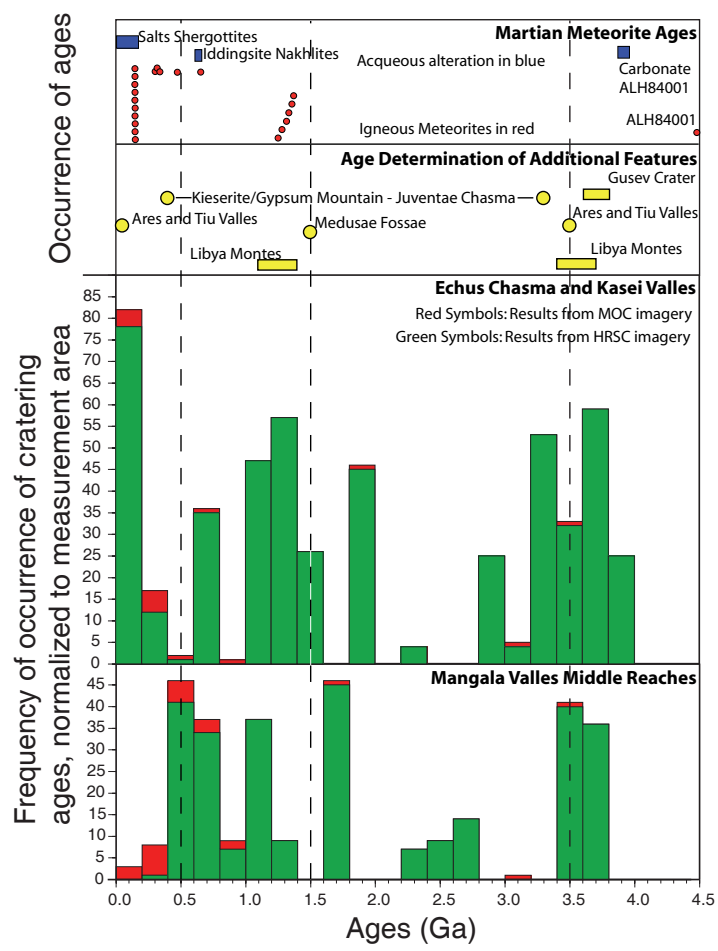

Fig. 2: Histogram of ages extracted from measurements on HRSC and MOC imagery in the Echus Chasma/Kasei Valles and Mangala Valles combined with cratering age measurements on additional features in comparison with known radiometric ages of martian meteorites (from [21]). 